\title{
Totalization of simplicial homotopy types
}

\author{
C. Ogle • A. Salch
}

Received: 8 May 2012 / Accepted: 23 May 2013 / Published online: 14 June 2013

(C) Tbilisi Centre for Mathematical Sciences 2013

\begin{abstract}
We identify the obstructions for the functoriality and the uniqueness of the totalization (or geometric realization) functor, (partially) defined on the category of simplicial objects in the homotopy category of a stable model category, and we use a result from the cyclic homology of group algebras to show they can be non-zero.
\end{abstract}

Keywords Homotopy category $\cdot$ Closed model category $\cdot$ Simplicial object

Mathematics Subject Classification (2000) Primary 18G55; Secondary 18G30 . $18 \mathrm{G} 35 \cdot 18 \mathrm{G} 40 \cdot 55 \mathrm{U} 10 \cdot 55 \mathrm{U} 15 \cdot 55 \mathrm{U} 35 \cdot 55 \mathrm{U} 40$

\section{Introduction/statement of results}

Let $\mathcal{C}$ denote a stable model category (our motivating example is the category of bounded-below chain complexes over $\mathbb{C}$ ). Then the associated homotopy category $H o(\mathcal{C})$ is triangulated in a natural way. Let $S_{\bullet} \mathcal{C}$ denote the category of simplicial objects over $\mathcal{C}$; the objects in this category are functors $F: \Delta^{o p} \rightarrow \mathcal{C}$, with morphisms given by natural transformations of such functors. Given such a simplicial object $C_{\bullet}=\left\{[n] \mapsto C_{n}\right\}_{n \geq 0}$, one can totalize it. (For our decision to refer to this construction as "totalization" and not its more common name, "geometric realization,"

Communicated by Paul Goerss.

C. Ogle $(\bowtie)$

Department of Mathematics, The Ohio State University, Columbus, OH 43210-1174, USA

e-mail: ogle@math.ohio-state.edu

A. Salch

Department of Mathematics, Wayne State University, Detroit, MI 48201-1347, USA 
see Remark 2.1.) We then have a totalization functor $T$ ot $: S_{\bullet} \mathcal{C} \rightarrow \mathcal{C}$. Using this functor, one has the following fundamental definition:

- A simplicial morphism $\phi_{\bullet}: C_{\bullet} \rightarrow D_{\bullet}$ is a weak equivalence in $S_{\bullet} \mathcal{C}$ iff $T$ ot $\left(\phi_{\bullet}\right)$ : $\operatorname{Tot}\left(C_{\bullet}\right) \rightarrow \operatorname{Tot}\left(D_{\bullet}\right)$ is a weak equivalence in $\mathcal{C}$.

This definition of weak equivalence yields a closed model structure on $S_{\bullet} \mathcal{C}$ compatible with that on $\mathcal{C}$ via the functor $T$ ot.${ }^{1}$ Now one can also consider the simplicial category $S_{\bullet} H o(\mathcal{C})$ of simplicial objects over the homotopy category, and two natural questions to ask regarding totalization are:

\section{Question 1 (Existence)}

a) (weak form) Let $\bar{C} \bullet$ be an object in $S_{\bullet} H o(\mathcal{C})$. Does $\operatorname{Tot}\left(\bar{C}_{\bullet}\right)$ exist? (If the answer is "yes," we will say that $\bar{C}$. is totalizable.)

b) (strong form) Let $\bar{C}_{\bullet}$ be an object in $S_{\bullet} H o(\mathcal{C})$. Does there exist an object $C_{\bullet}$ of $S_{\bullet} \mathcal{C}$ with $\bar{C}_{\bullet}=\left[C_{\bullet}\right]$ ?

This issue has been partially addressed by Bökstedt and Neeman in [1]. Precisely, in $[1, \S 3]$, the authors show that the natural construction of $\operatorname{Tot}\left(\bar{C}_{\bullet}\right)$ as a homotopy colimit in the (triangulated) homotopy category $\mathrm{Ho}(\mathcal{C})$ can be realized iff a sequence of first-order Toda brackets vanishes (cf. [5]). In fact their argument shows a bit more, so we recall their setup. Let $\bar{C}_{*}$ denote the chain complex object in $H o(\mathcal{C})$ associated to $\bar{C}$. by taking alternating sums of face sums (this constructions makes sense since triangulated categories are additive, so we can add and subtract the face maps from each other). For each $n \geq 0$, let $T_{n}$ denote the (hypothetical) total object of the "nskeleton" $\bar{C}_{n} \rightarrow \bar{C}_{n-1} \rightarrow \cdots \rightarrow \bar{C}_{0}$. Obviously $T_{0}=\bar{C}_{0}$, and $T_{1}$ is the mapping cone of $\bar{C}_{1} \rightarrow \bar{C}_{0}$, described by the triangle

$$
\bar{C}_{1} \rightarrow \bar{C}_{0} \rightarrow T_{1} \rightarrow \Sigma \bar{C}_{1}
$$

The two nulhomotopies of the composition $\bar{C}_{2} \rightarrow \bar{C}_{1} \rightarrow \bar{C}_{0} \rightarrow T_{1}$ allow one to define a map $\Sigma \bar{C}_{2} \rightarrow T_{1}$. We let $T_{2}$ be the mapping cone of this map. One then considers the composite $\phi_{3}: \Sigma \bar{C}_{3} \rightarrow \Sigma \bar{C}_{2} \rightarrow T_{1}$, which is the first Toda bracket associated to the homotopy chain complex $\bar{C}_{*}$. If $\phi_{3}=0$, then we get a map $\Sigma^{2} \bar{C}_{3} \rightarrow T_{2}$, and we let $T_{3}$ be its mapping cone. Then one considers the composite $\phi_{4}: \Sigma^{2} \bar{C}_{4} \rightarrow \Sigma^{2} \bar{C}_{3} \rightarrow T_{2}$. If $\phi_{4}=0$, then we get a map $\Sigma^{3} \bar{C}_{4} \rightarrow T_{3}$, and we let $T_{4}$ be its mapping cone. At this point the inductive construction should be clear. The argument of [1] leads to the following, which essentially answers part a) of Question 1.

Theorem [1] Let $\bar{C}_{*}, T_{i}, i=0,1$ be as above. Suppose that for $k<n, T_{k}$ exists and that there is a triangle

$$
T_{k-1} \rightarrow T_{k} \rightarrow \Sigma^{k} \bar{C}_{k} \rightarrow \Sigma T_{k-1}
$$

\footnotetext{
1 This is in distinction to [8], where Reedy shows that the category of simplicial objects over a model category admits a model structure when using the more restrictive notion of degreewise weak equivalence.
} 
Then the $(n-2)$ nd Toda bracket associated to the complex $\bar{C}_{*}$

$$
\phi_{n}: \Sigma^{n-2} \bar{C}_{n} \rightarrow T_{n-1}
$$

is defined, and $T_{n}$ can be constructed from this data iff $\phi_{n}=0$. The totalization $T_{\infty}$ exists iff $T_{n}$ can be constructed in this fashion for each $n \geq 0$, in which case one has $T_{\infty}:=\underset{h o c o l i m}{\rightarrow} T_{n}$.

Thus the total complex $T_{\infty}$ of $\bar{C}_{*}$, if it exists, is naturally equipped with a skeletal filtration $\left\{T_{n}\right\}$, for which the associated graded object satisfies $G r_{n}\left(T_{\infty}\right) / G r_{n-1}\left(T_{\infty}\right)=$ $\Sigma^{n} \bar{C}_{n}$ for each $n$. Note that this construction does not resolve the existence issue raised by part b), for which the obstructions seem to be a bit more subtle. Nevertheless, following this train of thought and motivated by the question posed by Bökstedt and Neeman on [1, p. 219], we have

Question 2a (Uniqueness) Let $C_{\bullet}, D_{\bullet}$ be two objects of $S_{\bullet} \mathcal{C}$, with the corresponding objects $\left\{[n] \mapsto\left[C_{n}\right]\right\}_{n \geq 0}$ resp. $\left\{[n] \mapsto\left[D_{n}\right]\right\}_{n \geq 0}$ in $S_{\bullet} H o(\mathcal{C})$ denoted by $\left[C_{\bullet}\right]$ resp. $\left[D_{\bullet}\right]$. If $\bar{f}_{\bullet}:\left[C_{\bullet}\right] \rightarrow\left[D_{\bullet}\right]$ is a simplicial map which is the identity in each degree, does $\bar{f}_{\bullet}$ extend to a filtration-preserving equivalence $g: \operatorname{Tot}\left(C_{\bullet}\right) \rightarrow \operatorname{Tot}\left(D_{\bullet}\right)$ ?

More generally, one can ask when a map of totalizable simplicial homotopy types induces a map of totalizations:

Question 2b (Functoriality) Let $C_{\bullet}, D_{\bullet}$ be two objects of $S_{\bullet} H o(\mathcal{C})$ whose totalizations both exist. If $f_{\bullet}: C_{\bullet} \rightarrow D_{\bullet}$ is a simplicial map, does $f_{\bullet}$ extend to a filtrationpreserving morphism $g_{*}: \operatorname{Tot}\left(C_{\bullet}\right) \rightarrow \operatorname{Tot}\left(D_{\bullet}\right)$ ?

As observed in $[1, \S 3]$, it is relatively easy to construct simplicial objects in $H o(\mathcal{C})$ for which the first possibly non-trivial Toda bracket is in fact non-trivial, from which one concludes that the totalization of $\bar{C}_{*}$ in general does not exist. There is, then, the secondary issue of the uniqueness of the totalization, framed as in Question 2a, and of functoriality, as in Question 2b. Our first main result is the identification of a series of obstructions associated to a morphism of simplicial objects in $\mathrm{Ho}(\mathcal{C})$ whose vanishing provides necessary and sufficient conditions for the existence of a filtrationpreserving morphism of totalizations to exist, as specified by these two questions. These obstructions may be thought of as the first and higher-order Toda brackets associated to a map of totalizable simplicial objects in the homotopy category $\mathrm{Ho}(\mathrm{C})$ (as defined below). In Sect. 3, we show that a well-known equivalence used to ompute the cyclic homology of group algebras, simplicialized, provides an explicit example of the non-triviality of these Toda brackets, and thus an explicit case of when the totalization, even when it exists, is not unique. Precisely, we show, for $\mathcal{C}$ the category of bounded-below chain complexes over $\mathbb{C}$ :

Theorem 1.1 There are simplicial chain complexes $C_{*, \bullet}, D_{*, \bullet}$ in $S_{*} \mathcal{C}$ such that

P1 There exists a morphism of graded chain complexes $\left\{\phi_{n}: C_{*, n} \rightarrow D_{*, n}\right\}_{n \geq 0}$ with $\phi_{n}$ a weak equivalence (i.e., quasi-isomorphism) for each $n$, 
P2 for each morphism $\alpha \in \operatorname{Hom}_{\Delta}([m],[n])$, there is a canonical chain homotopy

$$
\phi_{m} \circ C_{*, \bullet}(\alpha) \simeq D_{*, \bullet}(\alpha) \circ \phi_{n}: C_{*, n} \rightarrow D_{*, m},
$$

$P 3$ and $H_{*}\left(\operatorname{Tot}_{*} C_{*, \bullet}\right) \neq H_{*}\left(\operatorname{Tot}_{*} D_{*, \bullet}\right)$.

We would like to thank the referee for bringing [2] to our attention, which considers issues related to the ones discussed here.

\section{Maps of simplicial homotopy types and higher Toda brackets}

Remark 2.1 The terminologically fastidious reader might insist that one totalizes a cosimplicial object, and one geometrically realizes a simplicial object. To that way of thinking, we should be writing "geometric realization" rather than "totalization" throughout this paper, since we are considering simplicial objects and not cosimplicial objects. The reason we have chosen to stick with the term "totalization" is that the main example in the last section of this paper, due to the first author, really involves a totalization in the most classical sense: a totalization of a double complex. A suitably modern statement of the Eilenberg-Zilber theorem is that there is a quasi-isomorphism between, on the one hand, the classical totalization of the alternating sum double complex of any simplicial chain complex of abelian groups; and on the other hand, the geometric realization of that same simplicial chain complex of abelian groups. So there is good precedent for our usage of the term "totalization" and it is motivated by our main example.

Now we come around to the main question of this paper: suppose $C_{\bullet}, D_{\bullet}$ are simplicial objects in the homotopy category $H o(\mathcal{C})$ of a stable model category $\mathcal{C}$. Suppose further that $C_{\bullet}, D_{\bullet}$ are both totalizable, that is, the totalizations of $C_{\bullet}$ and $D_{\bullet}$ in the sense of Theorem 1 both exist. Finally, suppose we have a map $f_{\bullet}: C_{\bullet} \rightarrow D_{\bullet}$ of simplicial objects in $H o(\mathcal{C})$. We sometimes call $f_{\bullet}$ a map of simplicial homotopy types. Does $f_{\bullet}$ induce a map of totalizations $T_{\text {ot }} C_{\bullet} \rightarrow \operatorname{Tot} D_{\bullet}$ ?

To $C$. we can associate an alternating sum chain complex object, for which we will write $C_{*}$. Let $\mathcal{F}_{*} C_{*}$ denote the skeletal filtration of $C_{*}$; that is, $\mathcal{F}_{n} C_{*}=$ $\left\{C_{k}\right\}_{0 \leq k \leq n}$, with $\mathcal{T}_{n} C_{*}:=\operatorname{Tot}\left(\mathcal{F}_{n} C_{*}\right)$. Finally, for $1 \leq l \leq n, n \geq 0$, let $G r_{n}^{l} \mathcal{F} C_{*}=\left(\mathcal{F}_{n} C_{*}\right) /\left(\mathcal{F}_{n-l} C_{*}\right)$; similarly for $D_{*}$. The object $\operatorname{Tot}\left(C_{*}\right)$ is filtered by $\left\{\operatorname{Tot}\left(\mathcal{F}_{n} C_{*}\right)\right\}_{n \geq 0}$, and for each $n$

$$
G r_{n}^{1} \mathcal{F} C_{*}=\left(\operatorname{Tot}\left(\mathcal{F}_{n} C_{*}\right)\right) /\left(\operatorname{Tot}\left(\mathcal{F}_{n-1} C_{*}\right)\right)=\Sigma^{n} C_{n} .
$$

By "filtration" here we mean we have a natural sequence of maps

$$
0 \simeq \operatorname{Tot}\left(\mathcal{F}_{-1} C_{*}\right) \rightarrow \operatorname{Tot}\left(\mathcal{F}_{0} C_{*}\right) \rightarrow \operatorname{Tot}\left(\mathcal{F}_{1} C_{*}\right) \rightarrow \operatorname{Tot}\left(\mathcal{F}_{2} C_{*}\right) \rightarrow \ldots
$$

whose homotopy colimit is $\operatorname{Tot}\left(C_{*}\right)=\operatorname{Tot}\left(C_{\bullet}\right)$. (We are writing $T$ ot for what is really a geometric realization, and this sequence of maps is sometimes called the geometric realization tower, a name which is more convincing if you draw the sequence vertically. 
We draw it this way in diagram 2.8, below.) Questions $2 \mathrm{a}$ and $2 \mathrm{~b}$ of the previous section may be reformulated as:

Question When does there exist a filtration-preserving homomorphism of totalizations $g_{*}: \operatorname{Tot}\left(C_{*}\right) \rightarrow \operatorname{Tot}\left(D_{*}\right)$ with $g_{n} \simeq f_{n}: G r_{n}^{1} \mathcal{F} C_{*} \rightarrow G r_{n}^{1} \mathcal{F} D_{*}$ for each $n$ ?

As we shall see, there is a naturally defined hierarchy of obstructions associated to the existence of such a map, and that even the first-order obstructions are in general non-zero. To describe them, first note that by Dold-Kan, the homotopy commutativity of the above diagram is equivalent to the statement that

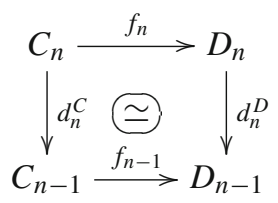

commutes in the homotopy category $H o(\mathcal{C})$ for each $n \geq 1$, with the vertical differentials given as the alternating sum of the face maps from dim. $n$ to dim. $n-1$. The first step to constructing $g_{\bullet}$ (or, equivalently, $g_{*}$ ) is provided by

Proposition 2.2 For each $n \geq 1$ there exists a filtration-preserving map

$$
\operatorname{Tot}\left(G r_{n}^{2} \mathcal{F} C_{*}\right) \stackrel{h}{\longrightarrow} \operatorname{Tot}\left(G r_{n}^{2} \mathcal{F} D_{*}\right)
$$

which on the subquotients $G r_{m}^{1} \mathcal{F} C_{*}$ agree with $f_{m}(m=n, n-1)$. If $f_{m}$ is a weak equivalence for every $m$, then so is $h$.

Proof Since Tot $\left(G r_{n}^{2} \mathcal{F} C_{*}\right)$ is just the cofiber of the map $d_{n+2}^{C}: \Sigma^{n+1} C_{n+2} \rightarrow$ $\Sigma^{n} C_{n+1}$, the map $h$ is just the map induced in cofibers:

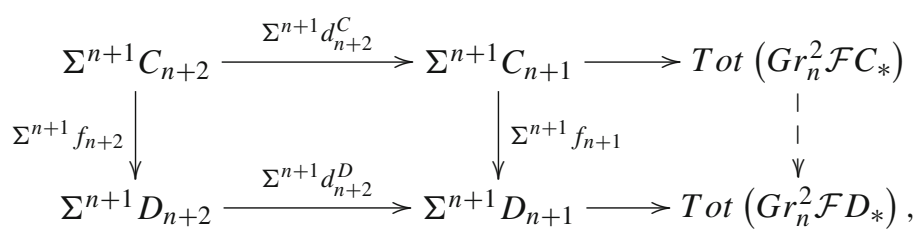

where the left-hand square commutes in $H o(\mathcal{C})$. That this map of cofibers exists is part of what one proves in the usual process of showing that the homotopy category of a stable model category is triangulated (or, more generally, that the homotopy category of a pointed model category is pretriangulated); one can consult Prop. 6.3.5 of [4].

We include an appealingly explicit construction of this map in the special case that $\mathcal{C}$ is the category of bounded-below chain complexes of $R$-modules, for some ring $R$. By assumption, for each $n$ there exists a chain homotopy $s(1)_{* n}: C_{* n} \rightarrow D[1,-1]_{* n}:=$ 
$D_{(*+1)(n-1)}$ with

$$
f_{* n-1} \circ d_{* n}^{C}-d_{* n}^{D} \circ f_{* n}=d_{(*+1)(n-1)}^{1 D} \circ s(1)_{* n}+s(1)_{(*-1) n} \circ d_{* n}^{1 C}
$$

where $d_{* m}^{1 C}: C_{* m} \rightarrow C_{(*-1) m}$ denotes the differential in the first coordinate (similarly for $\left.D_{* *}\right)$. Now $\operatorname{Tot}_{k+n}\left(\mathcal{F}_{n} C_{* *} / \mathcal{F}_{n-2} C_{* *}\right) \cong C_{(k+1)(n-1)} \oplus C_{k n}$ and similarly for $D_{* *}$. By equation (2.2) above, the map

$$
\begin{array}{r}
C_{(k+1)(n-1)} \oplus C_{k n} \rightarrow D_{(k+1)(n-1)} \oplus D_{k n}, \\
\left(x_{1}, x_{2}\right) \mapsto\left(f_{(k+1)(n-1)}\left(x_{1}\right)+s(1)_{k n}\left(x_{2}\right), f_{k n}\left(x_{2}\right)\right)
\end{array}
$$

defines a chain map of total complexes $T o t_{*}\left(G r_{n}^{2} \mathcal{F} C_{* *}\right) \rightarrow T o t_{*}\left(G r_{n}^{2} \mathcal{F} D_{* *}\right)$ which, by construction, agrees with $f_{\bullet}$ on the subquotients $G r_{m}^{1} \mathcal{F} C_{* *}$ for $m=n, n-1$.

Now since the square

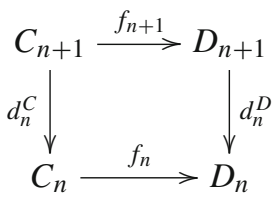

commutes up to homotopy, the difference $f_{n} \circ d_{n}^{C}-d_{n}^{D} \circ f_{n+1}$ is a nulhomotopic map $C_{n+1} \rightarrow D_{n}$. Of course, the same is true with $n$ replaced by $n+1$ throughout. As a consequence, in the diagram

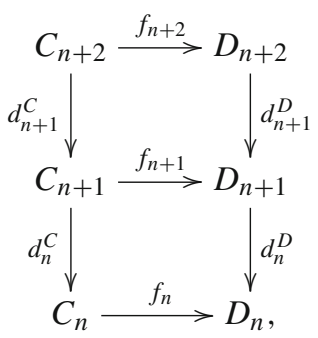

we have two nulhomotopies of the difference map

$$
f_{n} \circ d_{n}^{C} \circ d_{n+1}^{C}-d_{n}^{D} \circ d_{n+1}^{D} \circ f_{n+2}: C_{n+2} \rightarrow D_{n},
$$

which specifies a map $T\left(2, n ; f_{\bullet}\right): \Sigma C_{n+2} \rightarrow D_{n}$. The homotopy class $\left[T\left(2, n ; f_{\bullet}\right)\right] \in\left[\Sigma C_{n+2}, D_{n}\right]$ is the obstruction to extending Proposition 2.2 to $G r_{n}^{3}$ :

Proposition 2.3 For each fixed $n \geq 2$ there exists a filtration-preserving weak equivalence

$$
\operatorname{Tot}\left(G r_{n}^{3} \mathcal{F} C_{*}\right) \stackrel{\simeq}{\longrightarrow} \operatorname{Tot}\left(G r_{n}^{3} \mathcal{F} D_{*}\right)
$$


agreeing with $f_{m}$ on the subquotients $G r_{m}^{1} \mathcal{F} C_{*}(m=n, n-1, n-2)$ iff $\left[T\left(2, n ; f_{\bullet}\right)\right]=$ 0 .

Proof We note that $\operatorname{Tot}\left(G r_{n}^{3} \mathcal{F} C_{*}\right)$ sits in a tower of cofiber sequences

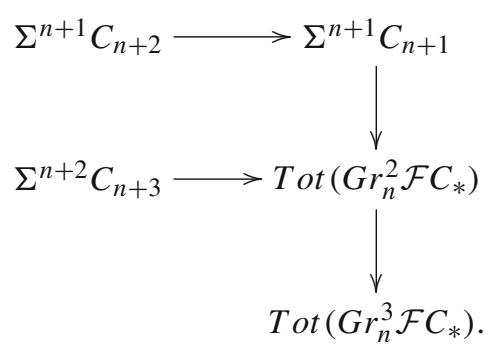

From Proposition 2.2, we know that we have a map defined up to homotopy on the top portion of this tower and its analogue for $D_{\bullet}$ :

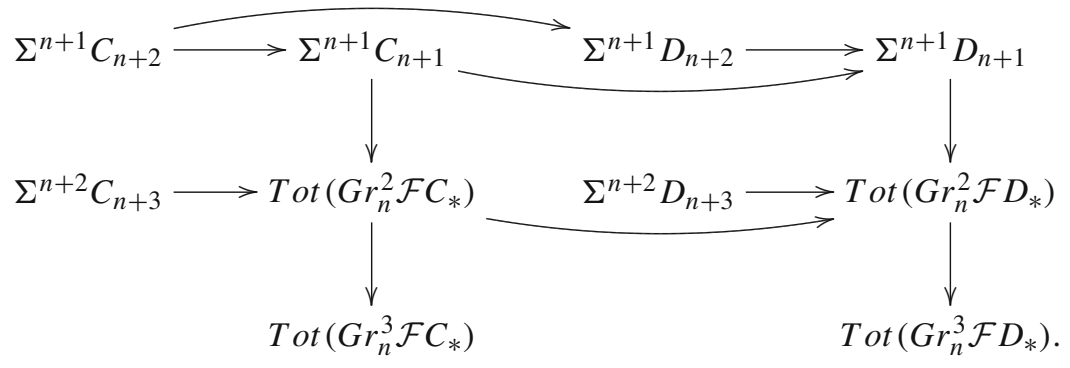

We want to extend this map, up to homotopy, to the bottoms of the towers. This is equivalent to asking that the square

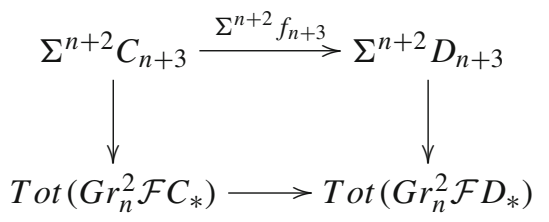

commute up to homotopy. Recall that the map $\Sigma^{n+2} C_{n+3} \rightarrow \operatorname{Tot}\left(G r_{n}^{2} \mathcal{F} C_{*}\right)$ arises from the two nulhomotopies of the map $\Sigma^{n+1} C_{n+3} \rightarrow \operatorname{Tot}\left(G r_{n}^{2} \mathcal{F} C_{*}\right)$, one arising from the nulhomotopy of the composite $\Sigma^{n+1} C_{n+3} \rightarrow \Sigma^{n+1} C_{n+2} \rightarrow \Sigma^{n+1} C_{n+1}$, and one arising from the nulhomotopy of the composite $\Sigma^{n+1} C_{n+2} \rightarrow \Sigma^{n+1} C_{n+1} \rightarrow$ $\operatorname{Tot}\left(G r_{n}^{2} \mathcal{F} C_{*}\right)$. Hence the homotopy-commutativity of diagram 2.5 is equivalent to the compatibility-up-to-homotopy of $f_{*}$ with these nulhomotopies, i.e., that the two nulhomotopies of $\Sigma^{n+1} C_{n+2} \rightarrow \Sigma^{n+1} D_{n+1}$ given by the two composites in the diagram 


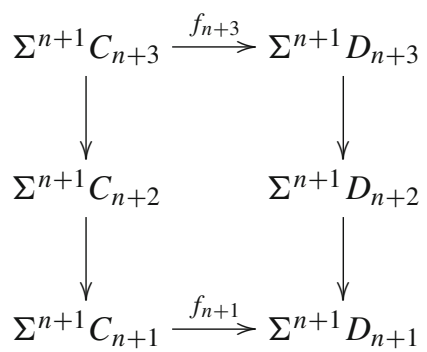

give rise to a nulhomotopic map $\Sigma^{n+2} C_{n+3} \rightarrow \Sigma^{n+1} D_{n+1}$. (That the two nulhomotopies of $\Sigma^{n+1} C_{n+2} \rightarrow \operatorname{Tot}\left(G r_{n}^{2} \mathcal{F} D_{*}\right)$ automatically give rise to the zero map $\Sigma^{n+2} C_{n+2} \rightarrow \operatorname{Tot}\left(G r_{n}^{2} \mathcal{F} D_{*}\right)$ is actually a restatement of Proposition 2.2!) But the map $\Sigma^{n+2} C_{n+3} \rightarrow \Sigma^{n+1} D_{n+1}$ in question is precisely $\Sigma^{n+1} T\left(2, n ; f_{\bullet}\right)$. Hence the vanishing of the Toda bracket $T\left(2, n ; f_{\bullet}\right)$ is equivalent to being able to extend the map on $G r_{n}^{2}$ to a map on $G r_{n}^{3}$.

The general case is described by the following theorem.

Theorem 2.4 Given a map of simplicial homotopy types $f_{\bullet}: C_{\bullet} \rightarrow D_{\bullet}$ as above, the map $f_{m}: G r_{m}^{1} \mathcal{F} C_{*} \stackrel{\simeq}{\longrightarrow} G r_{m}^{1} \mathcal{F} D_{*}$ extends to a filtration-preserving map

$$
\operatorname{Tot}\left(G r_{n}^{k} \mathcal{F} C_{*}\right) \longrightarrow \operatorname{Tot}\left(G r_{n}^{k} \mathcal{F} D_{*}\right)
$$

for some fixed $k \geq 3$ iff the Toda brackets $T\left(N, i ; f_{\bullet}\right)$ vanish for all pairs of integers $(N, i)$ with $2 \leq N<k$ and $n \leq i$ and $N+i<n+k$. Given this vanishing, the next higher-order set of Toda brackets are defined:

$$
T\left(k, n ; f_{\bullet}\right): \Sigma^{k-1} C_{n+k} \rightarrow D_{n}
$$

is the map given by the two nulhomotopic maps in the square

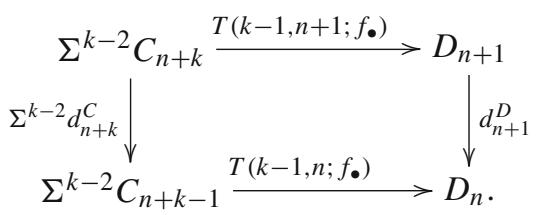

If the Toda brackets vanish for all orders and degrees, then there exists a filtrationpreserving map

$$
\operatorname{Tot}\left(C_{*}\right) \longrightarrow \operatorname{Tot}\left(D_{*}\right)
$$

which on $G r_{\bullet}^{1} \mathcal{F} C_{*}$ agrees with $f_{\bullet}$.

Finally, if $f_{n}$ is a weak equivalence for each $n$, then the map on $\operatorname{Tot}\left(G r_{m}^{k} \mathcal{F} C_{*}\right)$ is also a weak equivalence whenever it is defined (i.e., whenever all the appropriate Toda brackets, described above, vanish).

Proof Essentially the same as in Proposition 2.3. We work by induction: assume the theorem is true for all $\ell \leq k$ for some $k$. In other words, assume that when $\ell \leq k$, the 
homotopy map $f_{\bullet}$ induces a map $\operatorname{Tot}\left(G r_{m}^{\ell} \mathcal{F} C_{*}\right) \rightarrow \operatorname{Tot}\left(G r_{m}^{\ell} \mathcal{F} D_{*}\right)$ if and only if all the Toda brackets $T\left(N, n ; f_{\bullet}\right)$ vanish for $2 \leq N<\ell$ and $n \leq i$ and $N+i<n+\ell$. We want to show that the same statement then holds for $k+1$. Under our assumptions, we have a partially-defined map of geometric realization towers:

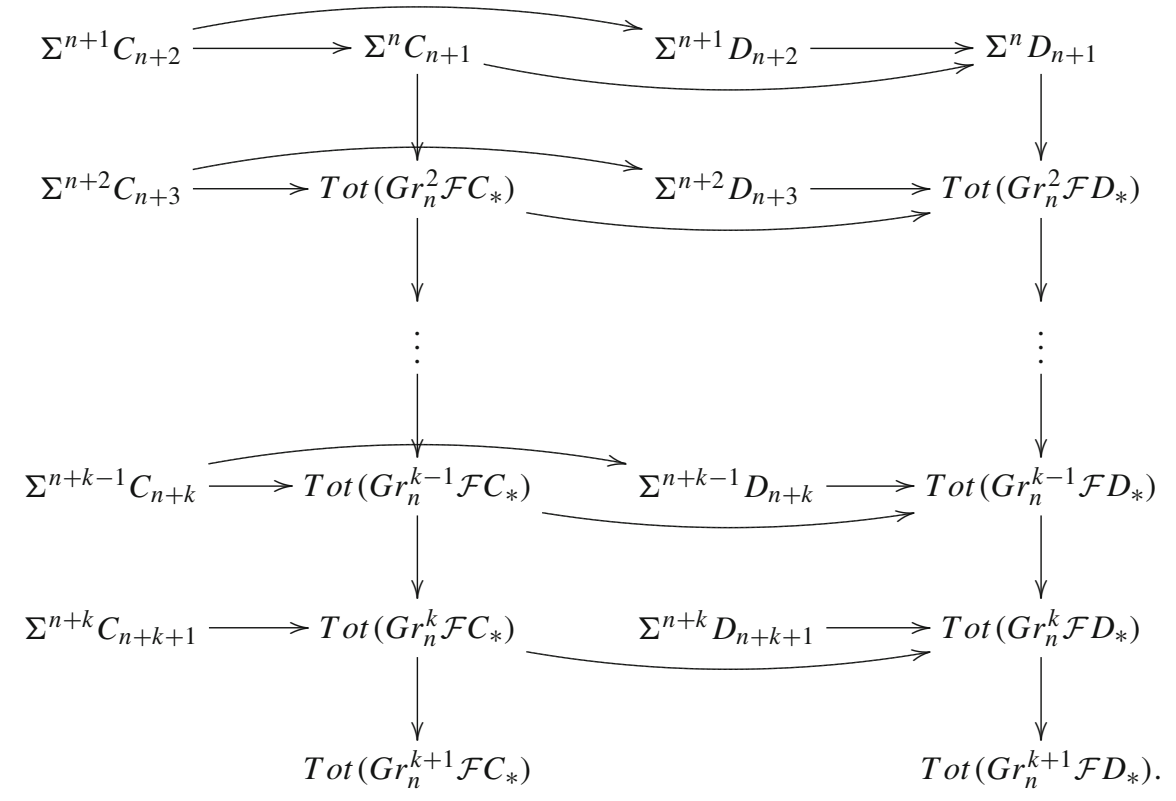

The problem of extending this map to the bottom-most stage of the towers is exactly the problem of extending the existing map from $\operatorname{Tot}\left(G r_{n}^{k} \mathcal{F} C_{*}\right) \rightarrow \operatorname{Tot}\left(G r_{n}^{k} \mathcal{F} D_{*}\right)$ to $\operatorname{Tot}\left(G r_{n}^{k+1} \mathcal{F} C_{*}\right) \rightarrow \operatorname{Tot}\left(G r_{n}^{k+1} \mathcal{F} D_{*}\right)$. The maps $\Sigma^{n+k} C_{n+k+1} \rightarrow \operatorname{Tot}\left(G r_{n}^{k} \mathcal{F} C_{*}\right)$ and $\Sigma^{n+k} D_{n+k+1} \rightarrow \operatorname{Tot}\left(G r_{n}^{k} \mathcal{F} D_{*}\right)$ at the bottoms of these towers arise from the two nulhomotopies of $\Sigma^{n+k-1} C_{n+k+1} \rightarrow \operatorname{Tot}\left(G r_{n}^{k} \mathcal{F} C_{*}\right)$ and the two nulhomotopies of $\Sigma^{n+k-1} D_{n+k+1} \rightarrow \operatorname{Tot}\left(G r_{n}^{k} \mathcal{F} D_{*}\right)$, and so the existence of the desired map at the bottom of the towers in diagram 2.6 is equivalent to the nulhomotopies of $\Sigma^{n+k-1} C_{n+k+1} \rightarrow \operatorname{Tot}\left(G r_{n}^{k} \mathcal{F} C_{*}\right)$ being compatible with the nulhomotopies of $\Sigma^{n+k-1} D_{n+k+1} \rightarrow \operatorname{Tot}\left(G r_{n}^{k} \mathcal{F} D_{*}\right)$, that is, it is equivalent to the two composite maps

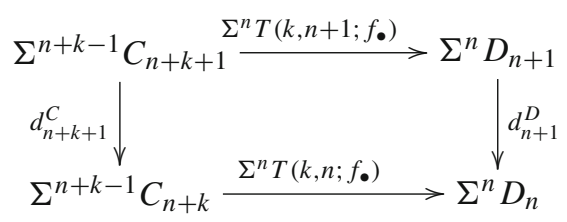

giving rise to a map $\Sigma^{n+k} C_{n+k+1} \rightarrow \Sigma^{n} D_{n}$ which is nulhomotopic. But this map $\Sigma^{n+k} C_{n+k+1} \rightarrow \Sigma^{n} D_{n}$ is precisely $\Sigma^{n} T\left(k+1, n ; f_{\bullet}\right)$. So the vanishing of the Toda bracket $T\left(k+1, n ; f_{\bullet}\right)$ occurs precisely when the partially-defined map of geometric realization towers in diagram 2.6 extends to a map $\operatorname{Tot}\left(G r_{n}^{k+1} \mathcal{F} C_{*}\right) \rightarrow$ $\operatorname{Tot}\left(G r_{n}^{k+1} \mathcal{F} D_{*}\right)$. 
We note that whenever a map of geometric realization towers as in diagram 2.6 exists, one notices that each induced map $\operatorname{Tot}\left(G r_{n}^{i+1} \mathcal{F} C_{*}\right) \rightarrow \operatorname{Tot}\left(G r_{n}^{i+1} \mathcal{F} D_{*}\right)$ in the tower is the map induced on cofibers of horizontal maps of a square

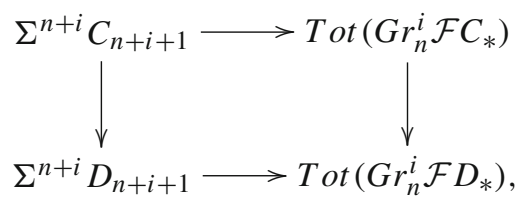

so if one knows that the vertical maps are weak equivalences, then so is the induced map $\operatorname{Tot}\left(G r_{n}^{i+1} \mathcal{F} C_{*}\right) \rightarrow \operatorname{Tot}\left(G r_{n}^{i+1} \mathcal{F} D_{*}\right)$. By an obvious induction we get that, if $f_{n}$ is a weak equivalence for each $n$, then so is each map $\operatorname{Tot}\left(G r_{n}^{i+1} \mathcal{F} C_{*}\right) \rightarrow$ $\operatorname{Tot}\left(G r_{n}^{i+1} \mathcal{F} D_{*}\right)$ whenever it is defined.

Now recall that one has both a homological and a cohomological spectral sequence associated to a tower of homotopy cofiber sequences. We start with the cohomological spectral sequence. Suppose one chooses an object $S$ of $\mathcal{C}$ and considers the representable functor $H:(H o(\mathcal{C}))^{o p} \rightarrow A b$ given by $H(-)=[-, S]$. This functor sends each triangle

$$
X \rightarrow Y \rightarrow Z \rightarrow \Sigma X
$$

in $H o(\mathcal{C})$ to a long exact sequence of abelian groups

$$
\cdots \rightarrow H(\Sigma X) \rightarrow H(Z) \rightarrow H(Y) \rightarrow H(X) \rightarrow H\left(\Sigma^{-1} Z\right) \rightarrow \cdots
$$

and, as a consequence, applying $H$ to the tower of homotopy cofiber sequences (i.e., triangles)

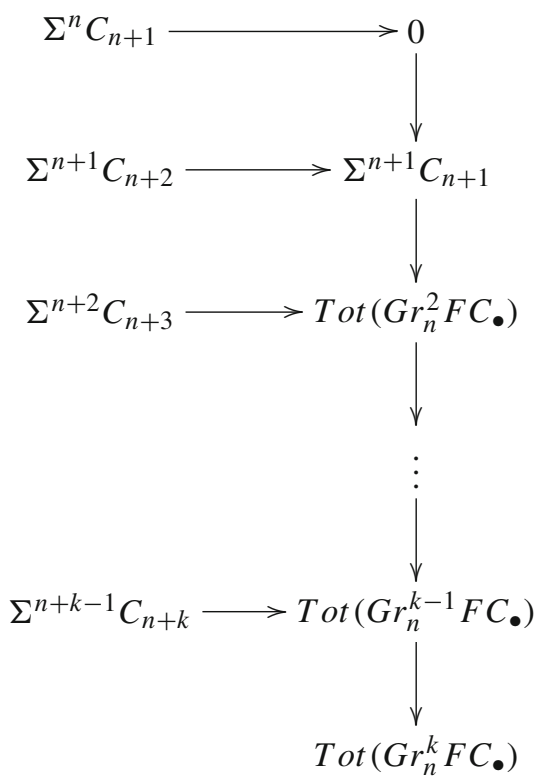


yields an exact couple and hence a spectral sequence. If we let $E_{1}^{s, t}=H\left(\Sigma^{t} C_{s}\right)$ if $n+$ $1 \leq s \leq n+k$ and 0 otherwise, then the associated spectral sequence has differentials

$$
d_{r}^{s, t}: E_{r}^{s, t} \rightarrow E_{r}^{s+r, t+r-1}
$$

and $d_{1}^{s, t}: H\left(\Sigma^{t} C_{s}\right) \rightarrow H\left(\Sigma^{t} C_{s+1}\right)$ coincides with the map $H\left(\Sigma^{t} d_{s+1}^{C}\right)$. The spectral sequence converges strongly to $H\left(\Sigma^{t-s} \operatorname{Tot}\left(G r_{n}^{k} F C_{\bullet}\right)\right)$.

If we instead have the infinite tower

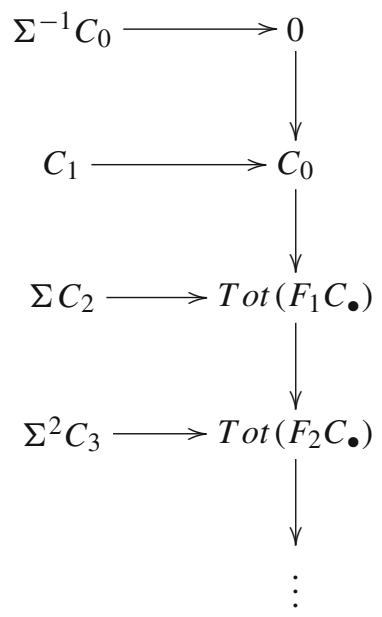

then our spectral sequence has $E_{1}^{s, t} \cong H\left(\Sigma^{t} C_{S}\right)$ and converges conditionally to $H\left(\Sigma^{t-s} \operatorname{Tot}\left(C_{\bullet}\right)\right)$.

We also have a homological spectral sequence. In order to get it to compute $\operatorname{Tot}\left(G r_{n}^{k} F C_{\bullet}\right)$ and not $\operatorname{Tot}\left(G r_{n}^{n} F C_{\bullet}\right) \simeq 0$, we first have to "dualize" the tower of diagram 2.7 by taking the levelwise homotopy cofiber of the map from that tower into the tower of homotopy cofiber sequences

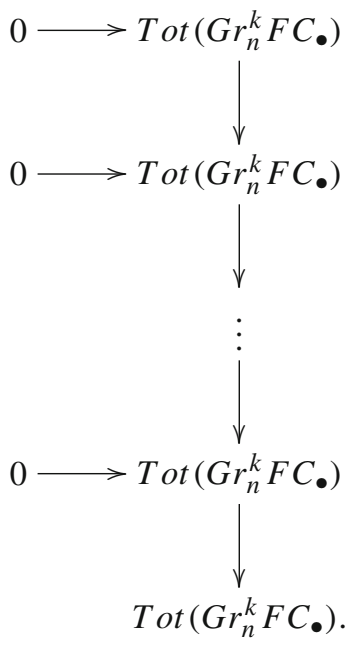


We write $T_{i}$ for the cofiber of the map $\operatorname{Tot}\left(G r_{n}^{i} F C_{\bullet}\right) \rightarrow \operatorname{Tot}\left(G r_{n}^{k} F C_{\bullet}\right)$. The levelwise cofiber tower we now have is the tower of homotopy cofiber sequences

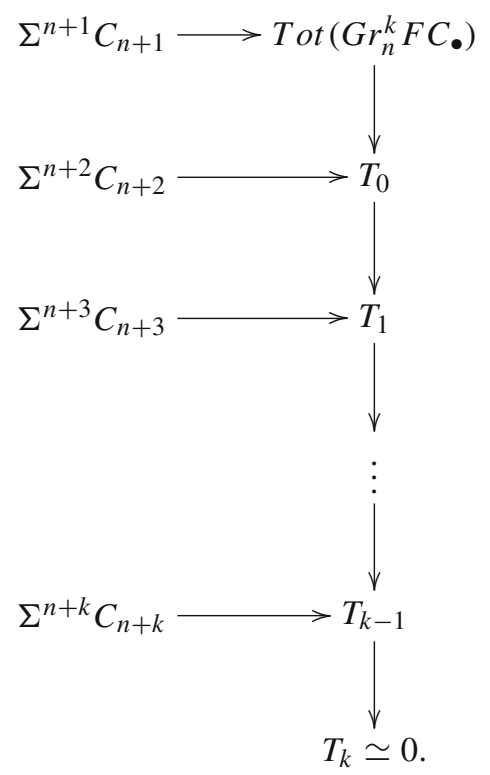

If $S$ is an object of $\mathcal{C}$, we have the co-representable functor $H: H o(\mathcal{C}) \rightarrow A b$ given by $H(-)=[S,-]$. Again, this functor sends triangles in $H o(\mathcal{C})$ to long exact sequences, so applying $H$ to the tower of homotopy cofiber sequences of diagram 2.9 yields an exact couple and hence a spectral sequence. If we let $E_{s, t}^{1}=H\left(\Sigma^{t} C_{s}\right)$ if $n+1 \leq s \leq n+k$ and 0 otherwise, then the associated spectral sequence has differentials

$$
d_{r}^{s, t}: E_{s, t}^{r} \rightarrow E_{s-r, t-r+1}^{r}
$$

and $d_{s, t}^{1}: H\left(\Sigma^{t} C_{s}\right) \rightarrow H\left(\Sigma^{t} C_{s-1}\right)$ coincides with the map $H\left(\Sigma^{t} d_{s}^{C}\right)$. The spectral sequence converges strongly to $H\left(\Sigma^{t-s} \operatorname{Tot}\left(G r_{n}^{k} F C_{\bullet}\right)\right)$. If we instead dualize the tower of diagram 2.8 and then apply $H$, the resulting spectral sequence has $E_{1}^{s, t} \cong$ $H\left(\Sigma^{t} C_{S}\right)$ and converges conditionally to $H\left(\Sigma^{t-s} \operatorname{Tot}\left(C_{\bullet}\right)\right)$.

The reason we are describing these spectral sequences is their relationship to the Toda brackets of Theorem 2.4. In the following theorem, in order to avoid having to write everything twice, we use the cohomological notation $E_{r}^{s, t}$ for our spectral sequence even though the theorem also applies equally well to the homological spectral sequence.

Theorem 2.5 Let $f_{\bullet}: C_{\bullet} \rightarrow D_{\bullet}$ be a map of totalizable simplicial homotopy types in $H o(\mathcal{C})$. Suppose $H$ is a cohomological functor $H(-)=[-, S]$ or a homological functor $H(-)=[S,-]$ on $H o(\mathcal{C})$, as above. Then we have the spectral sequences

$$
E_{1}^{s, t} \cong H\left(\Sigma^{t} C_{s}\right) \Rightarrow H\left(\Sigma^{t-s} \operatorname{Tot}\left(C_{\bullet}\right)\right)
$$


and

$$
E_{1}^{s, t} \cong H\left(\Sigma^{t} D_{s}\right) \Rightarrow H\left(\Sigma^{t-s} \operatorname{Tot}\left(D_{\bullet}\right)\right)
$$

The map $f_{\text {. }}$ induces a map from the $E_{1}$-page of spectral sequence 2.10 to the $E_{1}$-page of spectral sequence 2.11. This map commutes with the differential $d_{1}$.

Suppose $k>2$ and the Toda brackets $T\left(N, i ; f_{\bullet}\right)$ vanish for all pairs of integers $(N, i)$ with $2 \leq N<k$ and $n \leq i$ and $N+i<n+k$. Then the map $f_{\bullet}$ induces a map from the $E_{r}$-page of spectral sequence 2.10 to the $E_{r}$-page of spectral sequence 2.11 for every $r<k$. This map commutes with the differential $d_{r}$.

Proof That $f_{\bullet}$ always induces a map on the $E_{1}$-pages of the spectral sequences is clear from the fact that the differential $d_{1}$ in these spectral sequences is precisely the differential in the alternating sum chain complex object for $C_{\bullet}$ or $D_{\bullet}$.

For the remaining claim in the statement of the theorem, we work by induction. Suppose $k>2$ and the Toda brackets $T\left(N, i ; f_{\bullet}\right)$ vanish for all pairs of integers $(N, i)$ with $2 \leq N<k$ and $n \leq i$ and $N+i<n+k$. Furthermore, suppose we know that this implies that $f_{\bullet}$ induces a map, for all $r<k-1$, from the $E_{r}$-term of the cohomological spectral sequence 2.10 to the $E_{r}$-term of the cohomological spectral sequence 2.11. We want to know that we then get an induced map on the $E_{k}$-terms which commutes with the differentials. (We work with the cohomological spectral sequences, but the proof for the homological spectral sequences in strictly dual.) Since the inductive hypothesis implies we have a well-defined map on the $E_{k-1}$-terms commuting with the differentials, on passing to cohomology we get a well-defined map of $E_{k}$-terms, and we must check that it commutes with the differentials. But $E_{k}^{s, t}$ in spectral sequence 2.10 is a subquotient of $H\left(\Sigma^{t} C_{s}\right)$ in which every element's image under the boundary map $H\left(\Sigma^{t} C_{s}\right) \rightarrow H\left(\Sigma^{t-s}\right.$ Tot $\left.F_{s} C_{\bullet}\right)$ lies in the image of the map $H\left(\Sigma^{t-s}\right.$ Tot $\left.F_{s+k-1} C_{\bullet}\right) \rightarrow H\left(\Sigma^{t-s} T\right.$ ot $\left.F_{s} C_{\bullet}\right)$, and the differential $d_{k}^{s, t}$ is just the composite

$$
E_{k}^{s, t} \rightarrow H\left(\Sigma^{t-s} \operatorname{Tot}_{s+k-1} C_{\bullet}\right) \rightarrow E_{k}^{s+k, t+k-1}
$$

where the right-hand map is induced by the map $\Sigma^{t+k-1} C_{s+k} \rightarrow \Sigma^{t-s}$ Tot $F_{S+k-1} C_{\bullet}$. The vanishing of $T\left(k, s ; f_{\bullet}\right)$ is precisely what we need in order to know that the square

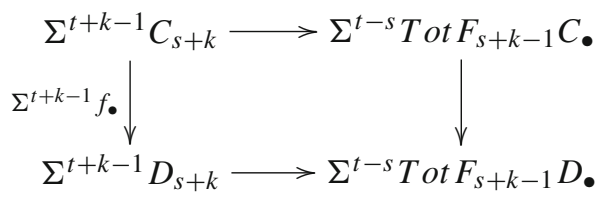

homotopy-commutes and hence that the induced map on $E_{k}$-pages commutes with the $d_{k}$ differentials. 


\section{Non-triviality of the obstruction}

For an algebra $A$, we write $C H_{*}(A)$ resp. $C C_{*}(A)$ for the Hochschild resp. cyclic complex of $A$, with conventions for the differentials and cyclic structure following that in [6]; $H H_{*}(A)$ resp. $H C_{*}(A)$ are their respective homology groups. When $A$ is the complex group algebra $\mathbb{C}[\pi]$, there are well-known decompositions of $C H_{*}(\mathbb{C}[\pi])$ and $C C_{*}(\mathbb{C}[\pi])$ as direct sums of subcomplexes, indexed on $<\pi>=$ the set of conjugacy classes of $\pi$, which induce corresponding decompositions in homology:

$$
\begin{aligned}
C H_{*}(\mathbb{C}[\pi]) \cong \bigoplus_{<x>\in<\pi>} C H_{*}(\mathbb{C}[\pi])_{<x>} \\
C C_{*}(\mathbb{C}[\pi]) \cong \bigoplus_{<x>\in<\pi>} C C_{*}(\mathbb{C}[\pi])_{<x>} \\
H H_{*}(\mathbb{C}[\pi]) \cong \bigoplus_{<x>\in<\pi>} H H_{*}(\mathbb{C}[\pi])_{<x>} \\
H C_{*}(\mathbb{C}[\pi]) \cong \bigoplus_{<x>\in<\pi>} H C_{*}(\mathbb{C}[\pi])_{<x>}
\end{aligned}
$$

Moreover, for each conjugacy class associated to an element of infinite order (or non-elliptic class), there are isomorphisms

$$
\begin{aligned}
& C H_{*}(\mathbb{C}[G])_{<x>} \cong C_{*}\left(B C_{x} ; \mathbb{C}\right) \\
& C C_{*}(\mathbb{C}[G])_{<x>} \cong C_{*}\left(B\left(C_{x} /(x)\right) ; \mathbb{C}\right)
\end{aligned}
$$

where $x$ is element representing the conjugacy class $\langle x\rangle, C_{x}$ denotes the centralizer of $x \in \pi$ and $(x) \subset C_{x}$ the infinite cyclic subgroup of $C_{x}$ generated by $x$. This identification, due to Burghelea [3], has been fundamental in understanding the structure of the Hochschild and cyclic homology groups of the group algebra. The isomorphism in (3.2) arises from the isomorphism in (3.1), which holds for all conjugacy classes. However, this identification involves a choice of element $x$ among the set of elements conjugate to $x$; as we shall see, it is impossible to make this choice in a way compatible, up to higher coherence homotopies, with respect to a collection of homomorphisms between two groups.

We recall how these equivalences are constructed. Given $x \in G$, write $S_{<x>}$ for the subset of elements in $G$ conjugate to $x$. There is a natural action of $G$ on $S_{<x>}$ given by $g \circ y:=g^{-1} y g$. We write $N^{c y}(G)$ for the cyclic bar construction on $G$; this is the cyclic simplicial set with

$$
\begin{aligned}
N^{c y}(G)_{n} & =G^{n+1} \\
\partial_{i}\left(g_{0}, \ldots, g_{n}\right) & =\left(g_{0}, \ldots, g_{i} g_{i+1}, \ldots, g_{n}\right), \quad 0 \leq i \leq n-1, \\
\partial_{n}\left(g_{0}, \ldots, g_{n}\right) & =\left(g_{n} g_{0}, g_{1}, \ldots, g_{n-1}\right), \\
s_{j}\left(g_{0}, \ldots, g_{n}\right) & =\left(g_{0}, \ldots, g_{j}, 1, g_{j+1}, \ldots, g_{n}\right)
\end{aligned}
$$


and cyclic structure given by

$$
t_{n}\left(g_{0}, \ldots, g_{n}\right)=\left(g_{n}, g_{0}, \ldots, g_{n-1}\right)
$$

There is a functorial equivalence

$$
C_{*}\left(N^{c y}(G) ; \mathbb{C}\right) \cong C H_{*}(\mathbb{C}[G])
$$

Moreover, the decomposition of $\mathrm{CH}_{*}(\mathbb{C}[G])$ into summands indexed on conjugacy classes arises from the decomposition of $N^{c y}(G)$ into a disjoint sum of path components

$$
N^{c y}(G) \cong \coprod_{<x>\in<G>} N^{c y}(G)_{<x>}
$$

where for each $\left\langle x>, N^{c y}(G)_{<x>}\right.$ is the simplicial subset of $N^{c y}(G)$ given by

$$
\left(N^{c y}(G)_{<x>}\right)_{n}:=\left\{\left(g_{0}, \ldots, g_{n}\right) \mid g_{0} g_{1} \cdots g_{n} \in S_{<x>}\right\}
$$

Denoting the non-homogeneous bar resolution of $G$ by $E G$, there is an isomorphism of simplicial sets (compare [6, Prop. 7.4.2])

$$
\begin{gathered}
S_{<x>} \underset{G}{\times} E G \stackrel{\cong}{\longleftrightarrow} N^{c y}(G)_{x}, \\
\left(g_{1} g_{2} \ldots g_{n} g_{0} ;\left[g_{1}, g_{2}, \ldots, g_{n}\right]\right) \leftrightarrow\left(g_{0}, g_{1}, \ldots, g_{n}\right)
\end{gathered}
$$

which sum together over conjugacy classes to induce a simplicial isomorphism

$$
S(G) \underset{G}{\times} E G \stackrel{\cong}{\longleftrightarrow} N^{c y}(G)
$$

where $S(G)=G$, but with $G$-action given by $g \circ s=g^{-1} s g, s \in S(G)$. Next, for any given element $y \in S_{<x>}$, there is an equivariant isomorphism of $G$-sets

$$
p_{y}: C_{y} \backslash G \stackrel{\cong}{\longrightarrow} S_{<x>}, \quad\left(C_{y}\right) g \mapsto g^{-1} y g
$$

This in turn induces an isomorphism of simplicial sets

$$
\left(C_{y} \backslash G\right) \underset{G}{\times} E G \stackrel{\cong}{\longleftrightarrow} S_{<x>} \underset{G}{\times} E G
$$

Finally, the inclusion $C_{y} \hookrightarrow G$ induces a weak equivalence

$$
B C_{y}=\left(C_{y} \backslash C_{y}\right) \underset{C_{y}}{\times} E C_{y} \stackrel{\simeq}{\hookrightarrow}\left(C_{y} \backslash G\right) \underset{G}{\times} E G
$$


The composition

$$
B C_{y} \rightarrow N^{c y}(G)_{<x>}
$$

is therefore a weak equivalence, as well as a map of cyclic simplicial sets, where the cyclic structure on the left is given by the "twisted nerve" construction detailed in [6, $\S 7.3 .3$ ] (in the notation of that source, we would write $B\left(C_{y}, y\right)$ instead of just $B C_{y}$ ). From this cyclic simplicial weak equivalence, one derives the usual identification of the non-elliptic summands in $C C_{*}(\mathbb{C}[G])$ as in (3.2).

With respect to naturality, a problem with this construction occurs in (3.5) and (3.6) where the choice of $y$ is made, since this choice cannot be done in a functorial way unless $\langle x\rangle=<i d>$. Fixing a choice of $y \in S_{<x>}$ amounts to choosing a basepoint for the non-basepointed discrete space $S_{<x>}$. In what follows, a free simplicial group refers to a simplicial group which is degreewise free.

Lemma 3.1 Suppose $\left(\Gamma_{\bullet}\right)$ is a free simplicial group. Then there is a natural map of graded simplicial sets

$$
\left\{[n] \mapsto \coprod_{<x>\in<\Gamma_{n}>} B C_{<x>}\right\} \stackrel{F\left(\Gamma_{\bullet}\right)}{\longrightarrow}\left\{[n] \mapsto \coprod_{n \geq 0} N^{c y}\left(\Gamma_{n}\right)_{<x>}\right\}_{n \geq 0}
$$

which, for each $n$ and $<x>\in<\Gamma_{n}>$, restricts to a weak equivalence of cyclic simplicial sets

$$
B C_{<x>} \stackrel{\simeq}{\hookrightarrow} N^{c y}\left(\Gamma_{n}\right)_{<x>}
$$

where $C_{<x>}$ is a canonical model for the centralizer subgroup $C_{x}$. Both the domain and range are simplicial spaces (i.e., bisimplicial sets), and for each iterated simplicial map $\lambda: \Gamma_{n} \rightarrow \Gamma_{m}$, there is a diagram

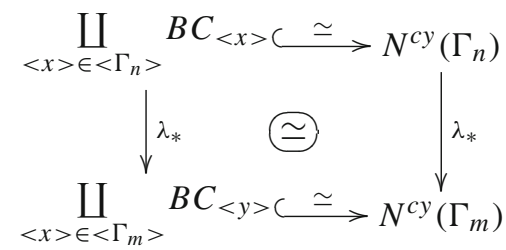

which commutes up to canonical homotopy

Proof For each $n \geq 0$ and conjugacy class $<x>\in<\Gamma_{n}>$, fix a choice of basepoint $x$ for $S_{<x>}$. We consider first the problem of constructing a canonical representative $C_{<y>}$ for the centralizer of an element $y$ when $<y>\neq<i d>$. If $<y>=<y^{\prime}>$, then choosing $h$ such that $y^{\prime}=y^{h}$ leads to an isomorphism $C_{y} \underset{\phi_{h}}{\cong} C_{y^{\prime}}$ given by $\phi_{h}(x)=x^{h}$. However, this isomorphic identification between the two centralizers is 
determined only up to precomposition with an inner automorphism of $C_{y}$, as the choice of $h$ is only determined up to right multiplication by an element of $C_{y}$. Thus a necessary and sufficient condition for $C_{<y>}$ to exist is that the group of inner automorphisms of $C_{y}$ is trivial; that is, $C_{y}$ should be abelian when $<y>\neq<i d>$. When $<y>\in<$ $F>, F$ a free group, $C_{y}$ is infinite cyclic for $y \neq i d$, so the condition holds. In fact, for $y \neq i d$, the centralizer $C_{y}$ is the infinite cyclic subgroup of $F$ generated by $x_{y}$, where $x_{y} \in F$ is uniquely defined as the element of shortest length for which $y$ can be written as a power of $x_{y}$, and the canonical isomorphism between $C_{y}$ and $C_{y^{\prime}}$ is the one that sends $x_{y}$ to $x_{y^{\prime}}$. Identifying $C_{y}$ with $C_{y^{\prime}}$ via this canonical isomorphism for conjugate elements $y$ and $y^{\prime}$ produces our canonical centralizer group $C_{<y>}$. We can extend this description to all conjugacy classes in $<F>$ by setting $C_{<1>}=C_{1}=F$.

There are maps of graded simplicial sets

$$
\begin{aligned}
& \left\{[n] \mapsto \coprod_{<x>\in<\Gamma_{n}>} B C_{<x>}\right\}_{n \geq 0} \stackrel{\simeq}{\longrightarrow}\left\{[n] \mapsto \coprod_{<x>\in<\Gamma_{n}>} C_{x} \backslash \Gamma_{n} \underset{\Gamma_{n}}{\times} E \Gamma_{n}\right\}_{n \geq 0} \\
& \underset{p_{*}}{\cong}\left\{[n] \mapsto \coprod_{<x>\in<\Gamma_{n}>} N^{c y}\left(\Gamma_{n}\right)_{<x>}\right\}_{n \geq 0}
\end{aligned}
$$

Where the first map in (3.10) is induced on each summand by the canonical isomorphism $C_{<x>} \cong C_{x}$ described above. For each $\partial_{i}: \Gamma_{n} \rightarrow \Gamma_{n-1}$ and $<x>\in<\Gamma_{n}>$ there is a commuting diagram

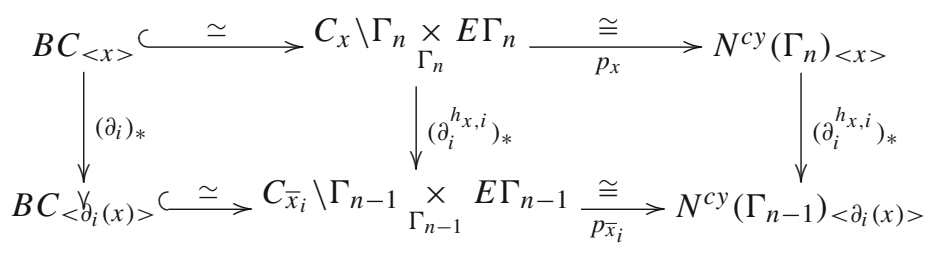

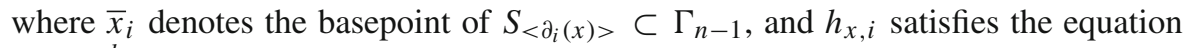
$\left(\partial_{i}(x)\right)^{h_{x, i}}=\bar{x}_{i}$. A similar diagram exists for degeneracy maps. Because of the conjugation by the $\left\{h_{x, i}\right\}$ the middle and right-most terms in the sequence of (3.10), equipped with conjugated face and degeneracy maps, may not be bisimplicial sets, but simply graded simplicial sets. However, from the preceding construction used in the definition of $C_{<x>}$, we see that the simplicial identities for compositions of face maps will be satisfied when restricted to the image of the inclusion of $B C_{<x>}$. The same argument applies for the other identities between compositions of face and degeneracy maps, and moreover the simplicial structure is independent of the particular choice of $\left\{h_{x, i}\right\}$. The result is a map of graded simplicial sets as indicated, which in each degree is a map of cyclic simplicial sets by [6, Prop. 7.4.5]. Iterating this construction for both face and degeneracy maps, one concludes that for any morphism $\alpha \in \operatorname{Hom}_{\Delta}([m],[n])$ 
and conjugacy class $\langle x\rangle$, there is a strictly commuting diagram

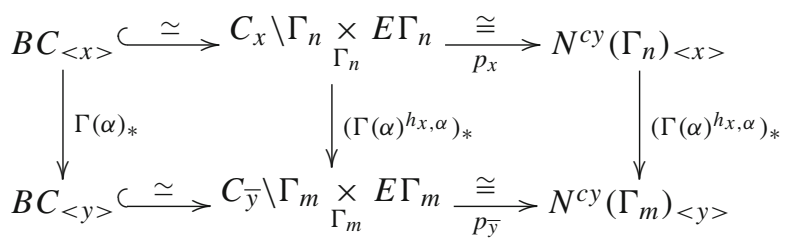

where $\Gamma(\alpha): \Gamma_{n} \rightarrow \Gamma_{m}$ is the homomorphism corresponding to $\alpha, y=\Gamma(\alpha)(x), \bar{y}$ is the basepoint of $S_{<y>}$, and $h_{x, \alpha} \in \Gamma_{m}$ satisfies the equation $(\Gamma(\alpha)(x))^{h_{x, \alpha}}=\bar{y}$. As conjugation by any element of $\Gamma_{m}$ induces a self map of $N^{c y}\left(\Gamma_{m}\right)$ canonically homotopic to the identity, we conclude the existence of a canonically homotopy commuting diagram

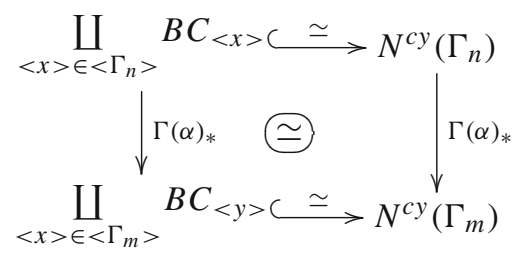

For a free group $F$, let

$\widetilde{C C}_{*}(\mathbb{C}[F]):=C_{*}(B F ; \mathbb{C}) \otimes C C_{*}(\mathbb{C}) \oplus \bigoplus_{<i d>\neq \neq x>\in<F>} C_{*}\left(B\left(C_{<x>} /(<x>)\right) ; \mathbb{C}\right)$

where $C_{<x>} /(<x>)$ denotes the canonical model for the centralizer of $x$ divided by the subgroup $(x)$. This chain complex is simply the cyclic chain complex (in char. $0)$ associated to the cyclic simpicial set $\coprod_{<x>\epsilon<F>} B C_{<x>}$. As we have seen in the proof of the previous Lemma, the association $F \mapsto \widetilde{C C}_{*}(\mathbb{C}[F])$ defines a functor (fr.gps) $\rightarrow \mathcal{C}$ from the category of free groups to $\mathcal{C}$.

Assume $m \geq 2, m$ even. Form a free simplicial group $\Gamma(m)$. by setting $\Gamma(m)_{j}=$ $\{i d\}$ for $j<m-1, \Gamma(m)_{m-1}=\mathbb{Z}$ on generator $\iota_{m-1}$, and $\Gamma_{m+k}$ the free group on generators $s_{\alpha}\left(\iota_{m-1}\right)$, where $s_{\alpha}$ ranges over iterated degeneracies from dim. $m-1$ to dim. $m+k$ when $k \geq 0$. This is a simplcial group model for $\Omega S^{m}$. Let $A(m)$. be the abelianization of $\Gamma(m) \bullet$, so that $|A(m) \bullet| \simeq K(\mathbb{Z}, m-1)$. As $m-1$ is odd, the simplicial group homomorphism $\Gamma(m) \bullet \rightarrow A(m)$. induced by abelianization is a rational homotopy equivalence, and the map of simplicial complex group algebras $\mathbb{C}\left[\Gamma(m)_{\bullet}\right] \rightarrow \mathbb{C}\left[A(m)_{\bullet}\right]$ a weak equivalence.

Define $C(m)_{*, \bullet}, D(m)_{*, \bullet}$ by

$$
\begin{aligned}
& C(m)_{*, \bullet}:=\widetilde{C C}_{*}\left(\mathbb{C}\left[\Gamma(m)_{\bullet}\right]\right) \\
& D(m)_{*, \bullet}:=C C_{*}\left(\mathbb{C}\left[\Gamma(m)_{\bullet}\right]\right)
\end{aligned}
$$


By the previous Lemma, both $C(m)_{*, \bullet}$ and $D(m)_{*, \bullet}$ are simplicial objects in $\mathcal{C}$, for which there is a homomorphism of graded complexes $\phi_{*, \bullet}: C(m)_{*, \bullet} \rightarrow D(m)_{*, \bullet}$ which is a quasi-isomorphism in each degree, and which commutes with face and degeneracy maps up to canonical chain homotopy. Degreewise inclusion of the summand indexed by $<i d>$ induces evident "assembly maps"

$$
\begin{aligned}
& H_{*}(K(\mathbb{Z}, m) ; \mathbb{C})=H_{*}\left(B \Gamma(m)_{\bullet} ; \mathbb{C}\right) \rightarrow H_{*}\left(C(m)_{*, \bullet}\right), \\
& H_{*}(K(\mathbb{Z}, m) ; \mathbb{C})=H_{*}\left(B \Gamma(m)_{\bullet} ; \mathbb{C}\right) \rightarrow H_{*}\left(D(m)_{*, \bullet}\right)
\end{aligned}
$$

Lemma 3.2 For all $m \geq 2, H_{m}\left(C(m)_{*, \bullet}\right) \neq H_{m}\left(D(m)_{*, \bullet}\right)$.

Proof Since the corresponding bicomplexes are positively graded in both coordinates, filtering by rows yields a strongly convergent spectral sequence

$$
\left\{E_{p, q}^{2}:=H_{p}\left(F_{*, q}\right) \Rightarrow H_{p+q}\left(F_{*, \bullet}\right)\right\}
$$

for $F=C, D$. In fact, the spectral sequences for both $C_{*, \bullet}$ and $D_{*, \bullet}$ have the same $E_{*, *}^{2}$-term. In both cases, the image of the canonical generator $\iota_{m} \in H_{m}(K(\mathbb{Z}, m) ; \mathbb{C})$ under the assembly map is represented at the $E^{1}$-level by the canonical generator $\iota_{1, m-1} \in E_{1, m-1}^{1} \cong H C_{1}\left(\mathbb{C}\left[\Gamma(m)_{m-1}\right]\right)=H C_{1}(\mathbb{C}[\mathbb{Z}]) \cong \mathbb{C}$. Moreover, in both cases, this element survives to a non-zero element in $E_{1, n-1}^{2}$. Now the bicomplex $C(m)_{*, *}$ satisfies the property that for each $n, 0=d_{1, n}^{0}: C(m)_{1, n} \rightarrow C(m)_{0, n}$. In other words, as a bicomplex it can be written as a direct sum $C_{*, *}=C_{0, *} \oplus C_{*, *} / C_{0, *}$. In the corresponding homology spectral sequence, this forces all differentials originating on the $q=0$ line to be zero. In particular, for the spectral sequence converging to $H_{*}\left(C(m)_{*, \bullet}\right)$, one has $0=d_{0, m+1}^{2}: E_{0, m+1}^{2} \rightarrow E_{1, m-1}^{2}$, implying $\iota_{1, n-1}$ survives to a non-zero element in $E_{1, m-1}^{3}=E_{1, m-1}^{\infty}$, so that $H_{m}\left(C(m)_{*, \bullet}\right) \cong \mathbb{C}$. On the other hand, in the spectral sequence converging to $H_{*}\left(D(m)_{*, \bullet}\right)$, the element $\iota_{1, n-1}$ must be hit by the differential $d_{0, m+1}^{2}$. In fact, $H_{*}\left(D_{*, \bullet}\right) \cong H C_{*}(\mathbb{C}[\Omega K(\mathbb{Z}, m)])$ by the above discussion (where $\Omega K(\mathbb{Z}, m)$ denotes any simplicial group rationally homotopy equivalent to $K(\mathbb{Z}, m))$. But for $m \geq 2, H C_{m}(\mathbb{C}[\Omega K(\mathbb{Z}, m)])=0$, with the canonical generator $0 \neq \iota_{m} \in H H_{m}(\mathbb{C}[\Omega K(\mathbb{Z}, m)])$ lying in the image of the $B$ : $H C_{m-1}(\mathbb{C}[\Omega K(\mathbb{Z}, m)]) \rightarrow H H_{m}(\mathbb{C}[\Omega K(\mathbb{Z}, m)])$ in the Connes-Gysin sequence.

Corollary 3.3 For each $m \geq 2$, the $(m+1)^{\text {st }}$ first-order Toda bracket associated to the homotopy chain map $C(m)_{*, \bullet} \rightarrow D(m)_{*, \bullet}$ is non-zero.

Proof This is an immediate consequence of Theorem 2.5.

Note that the above phenomenon is fundamentally a non-commutative one. In fact, revisiting the proof of Lemma 3.1 we see that the canonical models for the centralizer subgroups are also defined when the simplicial group is degreewise abelian. However, in this case, the diagram in (3.9) commutes not just up to canonical homotopy, but on the nose. In other words, if $A_{\bullet}$ is a simplicial abelian group, there is a homomorphism 
of simplicial chain complexes (not just graded complexes)

$$
\left\{[n] \mapsto \widetilde{C C}_{*}\left(\mathbb{C}\left[A_{n}\right]\right)\right\}_{n \geq 0} \rightarrow\left\{[n] \mapsto C C_{*}\left(\mathbb{C}\left[A_{n}\right]\right)\right\}_{n \geq 0}
$$

(with the left-hand side defined exactly as above) which is a quasi-isomorphism in each degree, hence a quasi-isomorphism of total complexes. From this we can also conclude that the functor

$$
\Gamma \bullet \mapsto \widetilde{C C}_{*}\left(\mathbb{C}\left[\Gamma_{\bullet}\right]\right)
$$

defined for simplicial groups which are either degreewise free or degreewise abelian, does not admit an extension to a homotopy functor from the category $S_{\bullet}(g p . s)$ of simplicial groups to $S_{\bullet} \mathcal{C}$, for this last observation implies that the abelianization map $\Gamma(m) \bullet \rightarrow A(m) \bullet$, which is a weak equivalence, does not induce a quasi-isomorphism when precomposed with $\widetilde{C C}_{*}(-)$.

\section{References}

1. Bökstedt, M., Neeman, A.: Homotopy limits in triangulated categories. Comp. Math. 86, 209-234 (1993)

2. Bousfield, A.K.: Homotopy spectral sequences and obstructions. Israel J. Math. 66, 54-104 (1989)

3. Burghelea, D.: The cyclic homology of the group rings. Comm. Math. 60, 354-365 (1985)

4. Hovey, M.: Model Categories, Mathematical Surveys and Monographs, vol. 63. American Mathematical Society, Providence (1999)

5. Kapranov, M.: On the derived categories of coherent sheaves on some homogeneous spaces. Inv. Math. 92, 479-508 (1988)

6. Loday, J.: Cyclic Homology, Grundlehren der mathematischen wissenschaften 301. Springer, Berlin (1992)

7. Quillen, D.: Homotopical Algebra, Lecture Notes in Math., vol. 43, Springer, Berlin-HeidelbergNew York (1967)

8. Reedy, C.: Homotopy theory of model categories, Preprint (1973) 